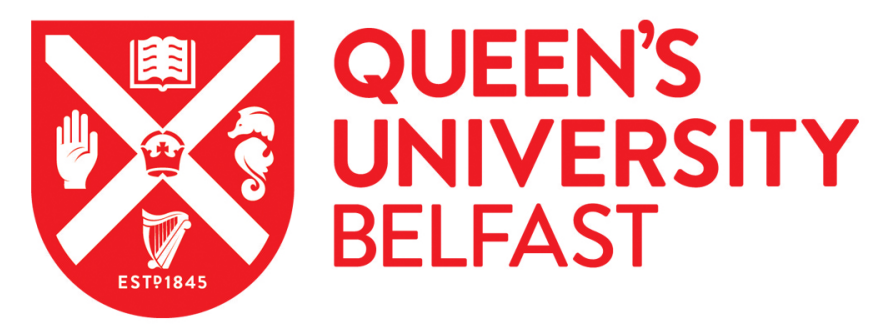

\title{
Interchange of entire communities: microbial community coalescence
}

Rillig, M. C., Antonovics, J., Caruso, T., Lehmann, A., Powell, J. R., Veresoglou, S. D., \& Verbruggen, E. (2015). Interchange of entire communities: microbial community coalescence. Trends in Ecology \& Evolution, 30(8), 470-476. https://doi.org/doi:10.1016/j.tree.2015.06.004

\section{Published in:}

Trends in Ecology \& Evolution

\section{Document Version:}

Peer reviewed version

Queen's University Belfast - Research Portal:

Link to publication record in Queen's University Belfast Research Portal

\section{Publisher rights}

(c) 2015, Elsevier. Licensed under the Creative Commons Attribution -NonCommercial-NoDerivs License

(https://creativecommons.org/licenses/by-nc-nd/4.0/), which permits distribution and reproduction for non-commercial purposes, provided the author and source are cited.

\section{General rights}

Copyright for the publications made accessible via the Queen's University Belfast Research Portal is retained by the author(s) and / or other copyright owners and it is a condition of accessing these publications that users recognise and abide by the legal requirements associated with these rights.

Take down policy

The Research Portal is Queen's institutional repository that provides access to Queen's research output. Every effort has been made to ensure that content in the Research Portal does not infringe any person's rights, or applicable UK laws. If you discover content in the Research Portal that you believe breaches copyright or violates any law, please contact openaccess@qub.ac.uk. 
2 Interchange of entire communities: microbial community coalescence

3 Matthias C. Rillig ${ }^{1,2^{*}}$, Janis Antonovics ${ }^{3}$, Tancredi Caruso ${ }^{4}$, Anika Lehmann ${ }^{1,2}$, Jeff R. Powell ${ }^{5}$,

4 Stavros D. Veresoglou ${ }^{1,2}$, Erik Verbruggen ${ }^{6}$

$5 \quad{ }^{1}$ Freie Universität Berlin, Institut für Biologie, Plant Ecology, D-14195 Berlin, Germany

$6{ }^{2}$ Berlin-Brandenburg Institute of Advanced Biodiversity Research (BBIB), D-14195 Berlin, Germany

$7 \quad{ }^{3}$ University of Virginia, Department of Biology, Charlottesville, Virginia VA 22904, USA

$8{ }^{4}$ School of Biological Sciences and Institute for Global Food Security, Queen's University of Belfast,

9 Belfast BT9 7BL, Northern Ireland, United Kingdom

10 Eniversity of Western Sydney, Hawkesbury Institute for the Environment, Richmond, NSW,

11 Australia

12 'University of Antwerp, Department of Biology, PLECO, Universiteitsplein 1 - 2610 Wilrijk, Belgium

13

$14 *$ Corresponding author: Rillig, M.C. (matthias.rillig@fu-berlin.de)

16 Keywords: communities; mixing; community coalescence; metacommunity; environment;

17 networks; biodiversity; global change 
Microbial communities are enigmatically diverse. We propose a novel view of processes likely

21 affecting microbial assemblages which could be viewed as the Great American Interchange en

22 miniature: the wholesale exchange among microbial communities resulting from moving pieces

23 of the environment containing entire assemblages. Incidental evidence for such 'community

24 coalescence' is accumulating, but such processes are rarely studied, likely because of the absence

25 of suitable terminology or a conceptual framework. We provide the nucleus for such a conceptual

26 foundation for the study of community coalescence, examining factors shaping these events,

27 links to bodies of ecological theory, and we suggest modeling approaches for understanding coalescent communities. We argue for the systematic study of community coalescence because of important functional and applied consequences.

Alfred Russell Wallace [1] was perhaps one of the first to consider what would happen when previously separated communities meet - in his case at a very large spatial and temporal scale, in what has become known as the Great American Interchange: the linking of North and South America by the appearance of the Isthmus of Panama. The result of such wholesale migration, 36 mixing and joining of communities was likely a multifold of establishments, species exchanges 37 and extinctions, massive effects at any rate. What if community encounter events like these were not exceptional singularities, but were to occur quite frequently, at time scales relevant to understanding community structure? Here we develop the idea that events reminiscent of the 
Interchange could be common in microbial systems, and with potentially even greater degrees

41 of mixing. We think this is the case because in nature, pieces of the environment much larger and

42 more persistent than an individual microbe, and indeed containing entire local microbial

43 communities, are routinely moved (see Figure 1 and Box 1). Forces that move pieces of the

44 environment containing entire microbial assemblages include gravity (falling leaves), animals

45 (e.g. burrows and casts), growth of macro-organisms (plant roots encountering each other in the

46 soil), wind (dust movement), flow in liquids (natural or industrial water bodies mixing, movement

47 inside the human body) or human activities (horticultural outplanting, ploughing or movement

48 of wood). We propose the term community coalescence (see Glossary) to describe such

49 community interchange events (Box 1).

50 While the literature is replete with studies on the effects and importance of many of the events

51 mentioned above (e.g. litter fall), these reports have rarely addressed the microbial community-

52 level interactions. The field of microbial biogeography (see Box 2) has long debated the degree

53 of dispersal limitation among micro-organisms, and is now also beginning to explore how

54 environment, spatial processes, and biotic context shape local communities. Community

55 coalescence events are part of the dispersal process, but also much more, because such events

56 result in whole communities and their environments interacting. Therefore, it is important to ask

57 how they influence the resulting community, and how these consequences can be linked to and

58 illuminated by existing ecological theory. What is the empirical evidence on community

59 coalescence thus far? How can the problem of entire communities interacting be approached

60 from a theoretical perspective? What are functional consequences of community coalescence?

61 These are the questions we address here with the goal of stimulating research on this topic; 
62 community coalescence is likely to be important not only in the everyday events of microbial

63 ecology, but also of increasing importance as the inter-connectedness of biological systems

64 increases with global change.

65

\section{Factors influencing community composition resulting from community coalescence}

67 While a number of empirical studies (see Box 1) and modelling exercises [2, 3] have addressed

68 aspects of what we call community coalescence, there has been no systematic study of such

69 phenomena, and this might in part be due to a lack of an applicable conceptual framework for

70 classifying these events, estimating their frequency, or predicting their consequences. We

71 believe such separate conceptual development is necessary because the coalescence of

72 communities has features quite distinct from those described in other bodies of theory, in

73 particular the processes envisaged in metacommunity theory [4]: (i) Metacommunity theory is

74 concerned with dispersal of individuals among local communities, and not with their wholesale

75 interchange. Thus in metacommunity theory, dispersal rate depends on the probability that local

76 communities (e.g. on islands) receive immigrants from the metacommunity (e.g. continent) while

77 coalescence is the encounter of entire local communities (e.g. an island community is

78 translocated to another island or to the continent). (ii) Community coalescence also allows for

79 and includes the movement and potential mixing of environments, for example aquatic

80 environments [5], and not just the movement of communities between environments. (iii)

81 Communities that coalesce do not necessarily belong to a metacommunity in the sense of

82 exchanging species at a low rate, and having their structure affected by such an exchange. 
83 Coalescence also occurs when there is physical relocation of habitats and accompanying changes

84 of the spatial structuring of the habitat. An example would be the encounter of the leaf microbial

85 communities and the soil biota; these would not normally be considered part of the same

86 metacommunity.

87 Community coalescence can occur in a number of ways (see Figure 2), and these interaction

88 modes could be captured by different types of theory. Coalescence events might differ in the

89 degree to which different environments are involved in the coalescence, including the creation

90 of 'mixed' environments or entry into one environment, the relative size of the interacting

91 communities (mixing ratios), the nature of the contact interface, and aspects of the temporal

92 nature of the coalescence events. Certain situations could then be grouped according to these

93 interaction modes in order to derive predictions for general rules; for example, in the kissing

94 situation the donated community would be experiencing the largely unaltered environment of

95 the recipient, the added community would be relatively small compared to the recipient

96 community, the contact interface would be a surface, and the interaction would be pulsed with

97 relatively short duration.

Some of these situations can be linked to existing bodies of ecological theory. For example, if one

99 community is moved to the environmental setting of another, environmental filtering [6] would

100 likely benefit the subset of species that already inhabited the recipient environment. In cases

101 where one community is added to another, priority effects [7] will be important, likely leading to

102 invasion-resistance of the recipient community. The number of individuals partaking in the

103 community transfer will also be important for the outcome; when the transferred communities 
104 include only a small subset of the component species, metacommunity theory [4] might become 105 more applicable.

106 It is likely that coalescing microbial communities will also contain predators and parasites [8], 107 such that the ecology of trophic interactions could be brought to bear on the problem. For 108 example, given that many predators are generalists, consuming a wide range of prey, the lack of 109 specific adaptations to predators could result in altered top-down control of the coalesced 110 community than in either of the two original communities. Conversely, during the coalescence, 111 specialist pathogens and consumers might suffer disproportionally, as the density of their hosts 112 would decline.

\section{Network theory and other approaches to model and analyze coalescent communities}

115 With these modes of interaction in mind, coalescence might be usefully modelled by using 116 network approaches. Interaction matrices describe the dynamics of every species as a function 117 of the other species, and in binary form the interaction matrix depicts the topology of 118 interactions, for example in a food web. In the context of community coalescence, we could 119 describe the topology of two (or more) initially non-interacting (e.g. physically separated) 120 admixed communities, and investigate the network topology resulting from coalescence. The 121 advantage of employing network theory to coalescence is that there is already a body of theory 122 pertaining to this situation in other areas of science $[9,10]$. Quite independently Kramer et al. $123[11]$ have used the term coalescence in the context of semi-independent neuronal networks that 124 under some circumstances start to interact. Synchronization of networks [9] is also potentially 
125 relevant to coalescence: when communities start to coalesce, there can be a transient period 126 during which they maintain their individual temporal patterns but eventually become 127 synchronized, with potentially destabilizing effects at the system level [12]. The study of temporal 128 fluctuations following a coalescence event can therefore yield key insights important for 129 understanding the assembly and stabilization of the resultant community. A fascinating aspect of 130 the study of temporal fluctuations in networks is that communities coming in contact with one 131 another maintain some internal temporal coherence (i.e. network modules [13]), at least for 132 some period of time. This coherence suggests that these communities interact as internally 133 integrated units rather than just as a collection of many species that suddenly interact with 134 another collection of species, resulting in a coalescent process where species replacement occurs 135 within these newly interacting integrated units rather than a more stochastic rearrangement of 136 interactions within the entire new network. This type of network dynamic is an emergent 137 property of the whole network, which arises from the fact that components of local communities 138 can in some cases act as whole units with strong interactions within these units and weak 139 interactions between [14]. We suggest that studying temporal synchronization [9, 10] in 140 coalescing microbial populations is an exciting multidisciplinary perspective with which to 141 document and understand such processes.

142 More classical network metrics such as (whole-network) modularity, connectance and 143 nestedness $[15,16]$ could also be used to describe networks in response to coalescence. 144 Interestingly, in network science modules are also called 'communities' [13] and can be 145 qualitatively defined as relatively dense subsets of vertices (i.e. species in our case) that are more 146 tightly connected internally than with the rest of the network. The quantitative definition of 
147 modules is not a trivial task (e.g. $[13,17])$ but by equating two interacting modules with two 148 coalescing communities, ecologists can exploit network tools to investigate coalescence in terms 149 of changes in network community structure. Furthermore, this type of interaction is not explicitly 150 considered in current ecological models describing community assembly from a metacommunity 151 perspective, possibly because in the last few decades community ecologists have often adopted 152 the implicit idea of communities as taxonomic assemblages or functional guilds at a given spatial 153 and temporal scale. In other words, community boundaries are generally defined based on the 154 unit that was sampled (whether an area or volume at a given time) as opposed to the interactions 155 occurring among members of the sample or the sampled units themselves. Therefore, the 156 identification of modules during the coalescent process and how these modules interact has the 157 potential to enhance ecological understanding at a profound level.

158 In this framework, expectations derived from simulations of coalesced communities [18-20] 159 could serve as null models for comparison of predicted with observed outcomes. Current work 160 on ecological networks (e.g. classical food webs) typically uses effects of single invasions or 161 species removals to derive estimates of how stable those networks are [16], but different results 162 might be expected when entire networks meet. Using model microbial communities with a 163 known network architecture would be one approach to test how the outcome of coalescence 164 varies with interaction frequency, size of communities, and environmental context (see Figure 2 165 and Box 1). 
166 Other tools might be helpful in characterizing the patterns emerging from coalescence as well.

167 The study of null models and $\beta$-diversity can be particularly promising [21-23], because changes 168 in $\beta$-diversity reflect the effects of immigration dynamics and biotic interactions.

169 However, the analysis of coalescent events presents new challenges that require the validation 170 of existing approaches and the development of new ones. For example, community dissimilarity

171 patterns across the landscape can provide insight into the extent that exchange between local

172 communities occurs and can be conceptualized in a metacommunity framework, [24]. But a 173 coalescence framework is needed to determine the degree to which admixing influences the 174 structure and functioning of the communities. There might be $a$ priori expectations based on the 175 original admixing communities where these can be identified and sampled; alternatively, 176 network analysis could allow accounting for their composition in an a posteriori fashion. 177 Importantly, however, we anticipate that coalescent events would result in complex outcomes 178 and that careful consideration will need to be given to whether existing common metrics or null 179 models can be used off-the-shelf or not [25].

\section{Potential consequences of coalescence for community-level functionality}

182 So far we have considered which factors might influence what communities resulting from 183 coalescence events might look like in terms of composition or network structure. However, it is 184 quite likely that community coalescence can also have strong functional consequences. We see 185 three main topics that should be the focus of future research: (i) degree of environmental mixing; 
186 (ii) dependence of functional consequences on traits of microbial species; and (iii) evolutionary

187 implications of coalescence (horizontal gene transfer and adaptations).

188 First, environmental mixing is explicitly included in the concept of community coalescence, and 189 therefore drastic changes in the environment during coalescence, i.e. changes in resources, 190 abiotic factors and biotic interactants, will also have to be considered. Some types of community 191 coalescence will lead to more drastic environmental changes with pervasive functional 192 consequences, such as the addition of communities to a new environment, with near-equal 193 mixing ratios (Fig. 2). Examples here would be predominantly from aquatic systems. Conversely, 194 environmental shifts will be least important for community coalescence events involving 195 relatively small additions of one community to another, with merely surfaces touching, and the 196 larger community remaining in its 'home' environment (like a quick kiss). Likely, any drastic 197 resource changes (e.g. nutrients and carbon) and altered abiotic conditions (e.g. $\mathrm{pH}$ and 198 temperature) will lead to species losses, for example by exceeding tolerances or via competitive 199 exclusion, with concurrent losses of functional traits from the resulting community.

200 The second aspect to consider is the trait space occupied by the communities prior to and after 201 coalescence (this is in part dependent on the discussion of environments above). Trait-based 202 approaches are increasingly being applied to microbial systems [26-28]. If the input communities 203 occupy quite dissimilar trait space from each other, and if these are partially maintained after 204 coalescence, then one could expect the resulting community to occupy an even wider trait space 205 than either of the original communities, likely also representing altered functionality [28], 206 including potentially greater productivity. The converse could be expected if input communities 
207 are quite similar in trait space: then members might merely substitute for each other in the 208 resulting community, not causing wholesale functional changes, with similar productivity.

209 The third point to consider are evolutionary implications of coalescence. Here we see two main 210 avenues for systematic study: horizontal gene transfer made possible by transient coexistence, 211 and adaptions to coalescence events.

212 Community coalescence could permit organisms from highly diverging habitats (e.g. river water 213 and soil) and with dissimilar traits to at least transiently coexist, especially with recurring events

214 (see Fig. 2). This co-occurrence can permit horizontal gene transfer between very dissimilar 215 organisms, an issue of considerable importance in trying to quantify, for example, the rates of 216 transfer of antibiotic resistance (e.g. [29]). As such these transient communities can be

217 'incubators' for rapidly-evolving species which exhibit different trait combinations, potentially 218 resulting in altered ecosystem functionality.

219 A second evolutionary question is whether frequent coalescence events in microbial 220 communities have brought about adaptations in member species, which in turn have functional 221 consequence at the community level. For example, many parasites have evolved life-cycle stages 222 in the gut of the host as well as in the soil, and the transfer of parasite stages between these 223 habitats occurs as a part of the coalescence of soil and gut communities (see Box 1). Another 224 example is the exchange of endophyte microbial communities cycling between leaves and the 225 soil. Do adaptations to coalescing communities occur in these and other microbial groups? 226 Predictions from evolutionary ecology on traits related to within- and between species 227 interactions are strongly contingent on levels of mixing (e.g. [30]): traits related to cooperative 
228 or mutually beneficial behavior are favored by spatial structure, and conversely 'selfish' non-

229 cooperative behaviors are favored in well-mixed systems. This is because this structuring 230 determines which individuals and/ or species are likely to iteratively interact over a prolonged

231 period of time, allowing cooperation to evolve. Given the propensity of positive interaction in

232 structured populations and communities, cycles of isolation followed by re-encounters in the

233 form of coalescence events might have a profound influence on the evolution of traits of the 234 constituent species and individuals.

\section{Applied relevance}

237 Microbial communities have an exceptional applied significance in many different fields from 238 medicine and biotechnology to environmental remediation and horticulture. It is likely that an 239 explicit consideration and conceptual treatment of coalescence involving microbial communities

240 inhabiting the human body, some of which are in intense exchange with the environment 241 (including other humans), could lead to a better understanding of their function in public health $242[29,31]$ and open new directions in biomedical research (e.g. gut microbiome interactions [32]); 243 in fact, some of the most intriguing empirical examples of coalescence so far stem from this area 244 of public health (see Box 1). Community coalescence could also be eminently applicable to 245 industrial processes, such as waste water treatment. Can community coalescence be used to 246 'engineer' microbial consortia [33] better suited to tasks than single communities? In agriculture, 247 the coalescence concept could help frame situations where substrates (e.g. biochar, manures, 248 compost and even crop seeds carrying endophyte communities) are added to resident soil 
249 microbial communities. In the context of global change and ever increasing connectedness of 250 global ecosystems, this concept can be very useful in capturing properties and dynamics of novel 251 communities and ecosystems [34]. But some of the most exciting potential applications are likely

252 to be completely unforeseeable until systematic study of these processes has commenced.

\section{Conclusion}

255 Our paper argues for the dedicated study of microbial community coalescence, which we 256 anticipate will address a set of new research questions (see Box 3). This will require a joint effort 257 from multiple disciplines and the empirical study of microbial communities that meet, of their

258 functional properties, as well as the development of models to simulate their dynamics and 259 evolution. Through this effort the concept of community coalescence can help better understand 260 the complexity of microbial assemblages and open avenues for the targeted manipulation of such 261 assemblages for human use in industry, medicine or environmental protection. While the 262 examples we have used are microbial in nature, we think that general insights derived from 263 microbial ecology might also be useful for understanding equivalent processes at larger 264 timescales in macro-organisms, especially given the context of ever increasing connectedness of 265 global ecosystems. 


\section{Glossary}

$\boldsymbol{\beta}$-diversity: the variation in the identities of species among sites [21].

Community coalescence: a joining of previously separate communities (or even ecosystems), forming a new entity that is not easily separable into parts again; this new entity has distinct properties from the parts it unites. The term 'coalescence' is also used in population genetics, but in a quite different context to indicate that homologous genes in different populations were at some point of necessity identical by descent, i.e. their history coalesces, and the genealogy of one gene is sometimes termed its 'coalescent'. This history is usually inferred from DNA sequence data.

Connectance: in ecological networks, the fraction of possible interactions that are actually realized.

Community: a general and broad term for any recognized assemblage of organisms containing multiple species that interact with one another due to their physical proximity.

Horizontal gene transfer: transfer of genes among unrelated species; postulated to occur by vectors such as viruses or insects, or by direct uptake of plasmids or environmental DNA.

Metacommunity: a collection of local communities linked by dispersal of their component species. The concept is derived from that of the metapopulation, which is a collection of populations of one species linked by dispersal of individuals. Metacommunity dynamics includes ecological 'rescue' of locally 'unfit' species, patch-dynamics (appearance and disappearance of 
287 habitat patches), extinctions and recolonizations from the regional species pool, and processes such as neutral drift (analogous to genetic drift) and species sorting (analogous to fitness differences).

Nestedness: in ecological networks, this measures the tendency for species with few links to exclusively interact with species with many links.

Network theory: describes interactions between multiple entities, which in ecology are typically species. Using network theory, communities can be described in terms of direct and indirect interactions among species. 


\section{Box 1. Evidence of microbial community coalescence}

Below we outline several previous studies that can be used to build a case that community coalescence occurs frequently and has important consequences.

Encounter and mixing of aquatic communities. - Souffreau et al. [35] and Adams et al. [36] present an experimental and observational investigation of bacterioplankton community interactions. In these studies communities encounter each other (e.g. river and lake) and are mixed in a partially new environment. Some of these encounters occur at very rapid rates, namely the flux rates of rivers, and at the level of entire assemblages. Livingston et al. [8] studied dynamics of aquatic communities which were experimentally mixed under controlled conditions; this study also explicitly included trophic interactions.

Interacting microbial communities in roots. - Mummey et al. [37] examined segments of roots growing in the immediate vicinity of roots of another plant species. The root-colonizing fungal communities were more similar to that of a heterospecific neighboring plant than the typical community of the species to which the root belonged. This was interpreted as propagules of one fungal community 'overwhelming' those of another. Hausmann and Hawkes [38] found similar effects in controlled pot experiments. Given that plant roots in communities typically intermingle, this coalescence of root-borne or rhizosphere microbial communities could be commonplace.

Microbial communities in the human body. - Qin et al. [39], using a metagenomic study of liver cirrhosis patients, found evidence for invasion of microbes from the mouth into the gut. A 
316 possible reason was a change in bile production in patients with disease, which permitted 317 invasion by bacteria from the oral microbiome. It thus seems that entire microbial communities, 318 occurring 'in series' in the digestive system, interact in complex ways and whose coalescence is 319 under metabolic or environmental control.

Transfer of oral microbial communities by kissing. - Kort et al. [40] studied the exchange of 321 bacteria after intimate kisses, including both observational and experimental data. They found substantial community exchange, leading to similarities among partners in oral microbiomes. Using tracer bacteria, the authors calculated an average transfer rate of 80 million bacteria per few-second kiss.

325 


\section{Box 2. Microbial biogeography}

In the past decade, there has been a resurgence of interest in microbial biogeography, and researchers have started to explicitly test and conceptualize whether 'biogeographical rules' also apply to microorganisms [41-44]. In this field, the major issue has been establishing the relative roles of dispersal limitation and environmental filtering as two fundamental factors that can shape microbial community turnover [45]. It has been argued that the potentially large dispersal distance of microbes precludes the 'existence of microbial biogeography' [46]. There is substantial variation among microbial taxa in dispersal capacity (e.g. $[47,48])$, and this debate, not surprisingly, has largely settled in favor of at least 'some' dispersal limitation shaping biogeography of microbes [49]. This has focused attention on the fact that interactions among microbes are likely potent causes of community variation and deserving of more attention (see because it considers how whole communities and their environments interact with each other and how this impacts on the dynamics of its members. 


\section{Box 3. Outstanding research questions}

Have repeated and continuous coalescence events contributed to the high microbial diversity in some habitats, like the soil?

Do 'hybrid' communities exhibit a broader functional range and higher productivity compared to communities entering a coalescence event?

Will members of communities with a history of coalescence have a higher persistence upon interaction with a 'naïve' community?

Can better mechanistic understanding of community coalescence help predict community-level migration and/ or mixing as a result of global change?

Can we predict properties of coalescent communities using approaches derived from the study of interacting networks?

Have microbes evolved specific adaptations to survive or profit from repeated coalescence events? What form do these adaptations take? Are alternative life-forms and complex life-cycles involved? When would antagonistic vs. mutualistic interactions be favored?

Is it possible to identify groups of microbes, or microbial traits, that are characteristic of coalescent events and can these be used as indicators of the coalescent history of a community? 


\section{$362 \quad$ Figure legends}

364 Figure 1. Encounters of entire microbial communities occur in many microbial systems. Examples

365 where such community coalescences are likely occur include (A, B) interaction of aquatic and 366 terrestrial systems, such as during flooding, in riparian zones or near ponds; (C) interaction of 367 communities inside the digestive system (e.g., oral and intestinal communities); (D) mixing of 368 communities inhabiting different water bodies in e.g. human-made industrial systems; (E) various 369 soil inputs, such as animal casts or leaves falling on the soil surface; (F) human-induced 370 movements of material, such as in horticulture or tree outplanting; (G) direct or indirect contact 371 between humans, such as two people kissing (also see Box 1). Photographs from MC Rillig (A, C, 372 D, E, F) and Wikimedia Commons (B: Niklas Tschöpe, G: anonymous).

375 Figure 2. Illustration of some factors that are likely to influence the outcome of community 376 coalescence. These factors include environmental conditions (entry of communities into new 377 environment vs. adding one community to another), the mixing ratios (equal vs. unequal 378 community proportions), the interaction interface (communities coalesce via surface touching 379 vs. wholesale mixing) or the temporal dynamics of community coalescence events (intermittent 380 pulses vs. regular exchange). General factors can be linked to existing bodies of ecological theory 381 (see text). 


\section{References}

3841 Wallace, A.R. (1876) The geographical distribution of animals. With a study of the relations of 385 living and extinct faunas as elucidating the past changes of the Earth's surface. Harper and

$386 \quad$ Brothers.

3872 Gilpin, M. (1994) Community-level competition-Asymmetrical dominance. Proc. Nat. Acad. Sci. $388 \quad$ U.S.A. $91: 3252-3254$

3893 Wright, C.K. (2008) Ecological community integration increases with added trophic complexity. 390 Ecol. Complex. 5: 140-145

3914 Leibold, M.A. et al. (2004) The metacommunity concept: a framework for multi-scale community 392 ecology. Ecol. Lett. 7: 601-613

3935 Bandelj, V. et al. (2012) Fuzziness and heterogeneity of benthic metacommunities in a complex $394 \quad$ transitional system. Plos One 7: e52395

3956 Kraft, N.J.B. et al. (2014) Community assembly, coexistence and the environmental filtering 396 metaphor. Funct. Ecol. DOI: 10.1111/1365-2435.12345

3977 Tucker, C.M. and Fukami, T. (2014) Environmental variability counteracts priority effects to 398 facilitate species coexistence: evidence from nectar microbes. Proc. R. Soc. B 281: 1-9

3998 Livingston, G. et al. (2013) The dynamics of community assembly under sudden mixing in $400 \quad$ experimental microcosms. Ecology 94: 2898-2906

4019 Arenas, A. et al. (2008) Synchronization in complex networks. Phys. Rep. 469: 93-153

40210 Munoz, M.A. et al. (2010) Griffiths phases on complex networks. Phys. Rev. Lett. 105: 1-4

$40311 \quad$ Kramer, M.A. et al. (2010) Coalescence and fragmentation of cortical networks during focal $404 \quad$ seizures. J. Neurosci. 30: 10076-10085

40512 Loreau, M. (2010) Linking biodiversity and ecosystems: towards a unifying ecological theory. 406 Philos. T. R. Soc. B. 365: 49-60 
40713 Girvan, M. and Newman, M.E.J. (2002) Community structure in social and biological networks.

$408 \quad$ Proc. Nat. Acad. Sci. U.S.A. 99: 7821-7826

40914 Krause, A.E. et al. (2003) Compartments revealed in food-web structure. Nature 426: 282-285

41015 Palla, G. et al. (2005) Uncovering the overlapping community structure of complex networks in $411 \quad$ nature and society. Nature 435: 814-818

41216 Pascual, M. and Dunne, J.A. (2006) Ecological networks: Linking structure to dynamics in food $413 \quad$ webs. Oxford University Press.

41417 Fortunato, S. (2010) Community detection in graphs. Phys. Rep. 486: 75-174

41518 James, A. et al. (2012) Disentangling nestedness from models of ecological complexity. Nature $416 \quad 487: 227-230$

41719 Pires, M.M. and Guimaraes, P.R., Jr. (2013) Interaction intimacy organizes networks of $418 \quad$ antagonistic interactions in different ways. J. R. Soc. Interface 10: 1-7

41920 Thebault, E. and Fontaine, C. (2010) Stability of ecological communities and the architecture of $420 \quad$ mutualistic and trophic networks. Science 329: 853-856

42121 Anderson, M.J. et al. (2011) Navigating the multiple meanings of beta diversity: a roadmap for the 422 practicing ecologist. Ecol. Lett. 14: 19-28

42322 Pigot, A.L. and Etienne, R.S. (2015) A new dynamic null model for phylogenetic community $424 \quad$ structure. Ecol. Lett. 18: 153-163

42523 Rosindell, J. et al. (2012) The case for ecological neutral theory. Trends Ecol. Evol. 27: 203-208

42624 Maurer, B.A. et al. (2013) Estimating metacommunity extent using data on species abundances, 427 environmental variation, and phylogenetic relationships across geographic space. Ecol. Inform. $428 \quad 13: 114-122$

42925 Gotelli, N.J. (2000) Null model analysis of species co-occurrence patterns. Ecology 81: 2606-2621 
43026 Aguilar-Trigueros, C.A. et al. (2015) Branching out: Towards a trait-based understanding of fungal ecology. Fungal Biol. Rev. DOI:10.1016/j.fbr.2015.03.001

43227 Aguilar-Trigueros, C.A. et al. (2014) Ecological understanding of root-infecting fungi using trait$433 \quad$ based approaches. Trends Plant Sci. 19: 432-438

$43428 \quad$ Krause, S. et al. (2014) Trait-based approaches for understanding microbial biodiversity and 435 ecosystem functioning. Front. Microbiol. 5: 1-10

43629 Mkrtchyan, H.V. et al. (2013) Could public restrooms be an environment for bacterial resistomes?

$437 \quad$ Plos One 8: e54223

43830 Lion, S. and van Baalen, M. (2008) Self-structuring in spatial evolutionary ecology. Ecol. Lett. 11: $439 \quad 277-295$

$44031 \quad$ Meadow, J.F. et al. (2014) Bacterial communities on classroom surfaces vary with human contact. $441 \quad$ Microbiome 2: 1-7

44232 Seedorf, H. et al. (2014) Bacteria from diverse habitats colonize and compete in the mouse gut. Cell 159: 253-266

44433 Shong, J. et al. (2012) Towards synthetic microbial consortia for bioprocessing. Curr. Opin. 445 Biotechnol. 23: 798-802

44634 Hobbs, R.J. et al. (2009) Novel ecosystems: implications for conservation and restoration. Trends $447 \quad$ Ecol. Evol. 24: 599-605

44835 Souffreau, C. et al. (2014) An experimental analysis of species sorting and mass effects in 449 freshwater bacterioplankton. Freshwat. Biol. 59: 2081-2095

45036 Adams, H.E. et al. (2014) Metacommunity dynamics of bacteria in an arctic lake: the impact of 451 species sorting and mass effects on bacterial production and biogeography. Front. Microbiol. 5: 1- 
$453 \quad 37$ Mummey, D.L. et al. (2005) Neighboring plant influences on arbuscular mycorrhizal fungal $454 \quad$ community composition as assessed by T-RFLP analysis. Plant Soil 271: 83-90

45538 Hausmann, N.T. and Hawkes, C.V. (2009) Plant neighborhood control of arbuscular mycorrhizal 456 community composition. New Phytol. 183: 1188-1200

45739 Qin, N. et al. (2014) Alterations of the human gut microbiome in liver cirrhosis. Nature 513: 59-64 $45840 \quad$ Kort, R. et al. (2014) Shaping the oral microbiota through intimate kissing. Microbiome 2: 41-41

$45941 \quad$ Bell, T. (2010) Experimental tests of the bacterial distance-decay relationship. Isme J. 4: 1357-1365

46042 Green, J.L. et al. (2004) Spatial scaling of microbial eukaryote diversity. Nature 432: 747-750

46143 Hanson, C.A. et al. (2012) Beyond biogeographic patterns: processes shaping the microbial 462 landscape. Nat. Rev. Microbiol. 10: 497-506

46344 Martiny, J.B.H. et al. (2006) Microbial biogeography: putting microorganisms on the map. Nat. $464 \quad$ Rev. Microbiol. 4: 102-112

$46545 \quad$ Baas Becking, L.G.M. (1934) Geobiologie of Inleiding tot de Milieukunde. W. P. van Stockum \& 466 Zoon.

46746 Finlay, B.J. (2002) Global dispersal of free-living microbial eukaryote species. Science 296: 1061$468 \quad 1063$

$46947 \quad$ Ingold, C.T. (1971) Fungal spores. Their liberation and dispersal. Clarendon Press.

47048 Young, K.D. (2006) The selective value of bacterial shape. Microbiol. Mol. Biol. Rev. 70: 660-703

47149 van der Gast, C.J. (2015) Microbial biogeography: The end of the ubiquitous dispersal hypothesis? $472 \quad$ Environ. Microbiol. 17: 544-546

47350 Dickie, I.A. et al. (2012) Do assembly history effects attenuate from species to ecosystem 474 properties? A field test with wood-inhabiting fungi. Ecol. Lett. 15: 133-141 